\title{
Blood perfusion construction for infrared face recognition based on bio-heat transfer
}

\author{
Zhihua Xie ${ }^{*}$ and Guodong Liu \\ Key Lab of Optic-Electronic and Communication, Jiangxi Sciences and Technology Normal \\ University, Nanchang 330013, China
}

\begin{abstract}
To improve the performance of infrared face recognition for time-lapse data, a new construction of blood perfusion is proposed based on bio-heat transfer. Firstly, by quantifying the blood perfusion based on Pennes equation, the thermal information is converted into blood perfusion rate, which is stable facial biological feature of face image. Then, the separability discriminant criterion in Discrete Cosine Transform (DCT) domain is applied to extract the discriminative features of blood perfusion information. Experimental results demonstrate that the features of blood perfusion are more concentrative and discriminative for recognition than those of thermal information. The infrared face recognition based on the proposed blood perfusion is robust and can achieve better recognition performance compared with other state-of-the-art approaches.
\end{abstract}

Keywords: Feature extraction, infrared image, face recognition, blood perfusion, bio-heat transfer model

\section{Introduction}

Due to its extensive application in identification and security, face recognition has attracted great attention of many researchers in computer vision and biometrics. Currently, most studies on face recognition focus on visual images [1]. However, the performance of visual face recognition is usually affected by variations in intrinsic (pose, expression, hairstyle. etc.) and extrinsic conditions (illumination, imaging system, etc.) [2]. Several studies have shown that the use of infrared images can solve the limitation of visible-image based face recognition, such as invariance to variations in illumination and robustness to variations in pose [3-5]. In addition, in recent years, the price of infrared cameras has decreased significantly, and their technology has improved with better resolution and quality, and the fixed pattern noise produced by old infrared cameras has been eliminated using non-uniformity correction techniques [6,7].

It is highlighted that the infrared images can be affected by ambient temperature, psychological and physiological conditions [4]. Therefore, the recognition systems based on thermal images will perform well when the test and train images are captured in the same ambient temperature, while the performance will be poor if the testing and training samples are collected under different temperature (timelapse data) $[8,9]$.

\footnotetext{
${ }^{*}$ Corresponding author: Zhihua Xie, Key Lab of Optic-Electronic and Communication, Jiangxi Sciences and Technology Normal University, Nanchang 330013, China. Tel.: 13970018047; Fax: 086-791-83831977; E-mail: xiezhihua@acm.org.
} 
Stable facial biological features extraction is vital to the improved performance of infrared face recognition under variable ambient temperatures, $[10,11]$. As we know, infrared images based on facial temperature depend on the distribution of blood vessels (DBV) and the velocity of blood perfusion (VBP), both of which are internal biological features hardly affected by the ambient atmosphere. Therefore, in terms of biology, they're more stable [2]. In this situation, many researchers make efforts to get the stable physiological information from the thermal images [12]. These efforts can be divided into two categories: blood vessels and blood perfusion under the skin from the thermal images [12]. M. Akhloufi et al. proposes a method of infrared face recognition reconstructing DBV [13]. For VBP, from the aspect of biomechanics, Wu et al. [11] founded a model converting temperature features into blood perfusion features based on the thermal equilibrium of the skin. This paper will focus on the latter.

The blood perfusion model proposed in the work of $\mathrm{Wu}$ et al. [11] improves the performance of infrared face recognition dramatically, especially for images in variable ambient temperatures. However, this model is based on the hypothesis of thermal equilibrium on the skin, instead of the heat conduction in skin tissues [12]. The model converts temperature into corresponding blood perfusion rate by the method of point-to-point regardless of the relativity of the neighbor positions in thermal image, which is in conflict with the heat conduction principle of skin tissues [14]. To address this problem, the classic bio-heat transfer equation (Pennes equation) is used to consider the relationship of the neighbor positions in thermal image. A new reconstruction method of blood perfusion is proposed. Discrete Cosine Transform (DCT) [15] and feature selection are combined to extract features for the final recognition results.

\section{Methods}

\subsection{Bio-heat \& Pennes equation}

Mathematical model is a theoretical foundation of bio-heat transfer. An ideal model can explain the mechanism of heat transfer in bio-tissue or between it and the environment, and play an important role in indicating the temperature field in tissues. In theory, bio-heat is about the conduction of heat and substance in tissues and the exchange of substance and energy between creatures and the environment $[11,14]$. It's Pennes' contribution in 1948 [16,17] which has distinguished heat conduction in tissues from that in common materials for the first time. It reduces forearms to cylinders, and takes into account blood perfusion in muscular tissues. Pennes proposes a bio-heat differential equation under axial symmetry:

$$
\rho c \frac{\partial T}{\partial t}=\nabla \cdot(k \nabla T)+W_{b} C_{b}\left(T_{a}-T\right)+q_{m}+q_{r}
$$

Here $\rho$ is the density of tissues, $c$ is the specific heat of tissues, $k$ is the coefficient of heat conduction for tissues, $W_{b}$ is the blood perfusion rate, $C_{b}$ is the specific heat of blood, $T$ is the temperature inside creatures, $T_{a}$ is the temperature of arterial blood, $q_{m}$ is the blood perfusion rate of heat produced in metastasis per stere (regarded as a constant in Pennes' model, $q_{m}=400\left(\mathrm{~J} / \mathrm{m}^{3} \cdot \mathrm{s}\right)$ ), $q_{r}$ is 
the perfusion rate of heat provided by the ambience. The parameters for the thermal characteristics and physiology of tissues are $\rho, c, k$ and $W_{b}, q_{m}$, respectively.

Pennes equation distinguishes itself from common heat conduction ones by a blood perfusion term:

$$
q_{b}=W_{b} C_{b}\left(T_{a}-T\right)
$$

where $W_{b}$ is the blood perfusion rate which will be analyzed discretely in this paper. This equation is a 3-dimensional elliptic partial differential equation for heat conduction of creatures. Since the proposition of Pennes equation, the equation has been improved by many researchers, such as Stolwijk, ChenHolmes, Weinbaum-Jiji, et al. [16,17]. All these equations take more physiological factors compared with Pennes equation, and describe the heat conduction of creatures more precisely. It is important to mention that those new bio-heat transfer models are based on the same bio-heat transfer principle. So the heat transfer process in the face can be modeled by the classic Pennes bio-heat transfer equation.

\subsection{Blood perfusion model based on Pennes equation}

The facial temperature field is 2-dimensional, while Pennes equation is 3-dimensional symmetric. To apply the Pennes equation to the facial temperature field, the 3-dimensional Pennes equation should be transformed into a 2-dimensional one. Under 2-dimensional thermal equilibrium, $\rho c \frac{\partial T}{\partial t}=0$. There's no heat source, so $q_{r}=0$. Moreover, facial tissues are isotropic. Thus, the original 3dimensional Pennes equation can be transformed into a 2-dimensional form as follows:

$$
K\left(\frac{\partial^{2} T}{\partial x^{2}}+\frac{\partial^{2} T}{\partial y^{2}}\right)+W_{b} C_{b}\left(T_{a}-T\right)+q_{m}=0
$$

Our main work in this section is to get blood perfusion rate by solving Eq. (3) reversely, given by the distribution of facial temperature field (facial thermograms)

$$
W_{b}=\frac{-K\left(\frac{\partial^{2} T}{\partial x^{2}}+\frac{\partial^{2} T}{\partial y^{2}}\right)-q_{m}}{C_{b}\left(T_{a}-T\right)}
$$

Infrared facial thermograms are discrete while Eq. (4) is continuous. In order to apply the continuous Pennes equation to infrared facial bio-heat transfer model, Pennes equation needs to be analyzed numerically. Let each pixel in given thermograms correspond to a blood perfusion rate, via digital Laplacian operator, the following formula can be obtained: 


$$
K\left(\frac{\partial^{2} T}{\partial x^{2}}+\frac{\partial^{2} T}{\partial y^{2}}\right)=K \frac{T(x-\nabla x, y)+T(x+\nabla x, y)+T(x, y-\nabla y)+T(x, y+\nabla y)-4 T(x, y)}{\nabla x * \nabla y}
$$

As our infrared facial images are captured by the same camera, the distance between adjacent pixels in an image is the same, namely $\nabla x=\nabla y=d$, where $d$ is the sample interval of discrete infrared faces. Eq. (4) can be expressed:

$$
W_{b}(i, j)=\frac{-K \lambda(T(i-1, j)+T(i+1, j)+T(i, j-1)+T(i, j+1)-4 T(i, j))-q_{m}}{C_{b}\left(T_{a}-T\right)}
$$

where $T(i, j)$ is the temperature, $\lambda=1 / d^{2}$ is determined by the interval of facial images. Faces are curved surfaces, while their infrared images are planes. Hence, there must be an error between intervals in real faces and those in infrared images. It means that $\lambda$ varies with different points. For convenience, we consider $\lambda$ to be a constant. Eq. (6) illustrates that the blood perfusion rate at a point is relevant with not only its own temperature, but also the temperature of its neighbors. This shows that the relation between blood and temperature is about the interaction between its neighbor points, instead of point-to-point. Therefore, our blood perfusion rate fits the bio-heat transfer principle better.

\subsection{Feature selection in DCT domain}

DCT is a popular image compression method [5], whose transformation nuclear is the real-time cosine function. Therefore, DCT has the properity of calculation simple and good information packing ability. Another merit of DCT is that it can be implemented efficiently using the Fast Fourier Transform (FFT). As a result, DCT can be applied to real-time features extraction [15].

For image compression, [18] makes use of the centralization of energy at the low-frequency region to select features of DCT coefficients. However, from the view of pattern recognition, centralization of energy doesn't mean that of discrimination, and some frequency components with low energy may contribute more to recognition than those with high energy [19]. Therefore, the traditional feature selection, Z-scan [18], is unsuitable for selecting effective discriminant features. To extract most suitable features for face recognition, this paper uses the separability discrimination $(S D)$ based on statistical learning to discriminative different DCT coefficients.

Suppose there're $C$ classes in training samples, each of which contains $S$ samples. For each DCT coefficient $A_{i j}$, where $i=0,1,2, \mathrm{~L}, M-1, j=0,1, \mathrm{~L}, N-1$, a 2-dimensional sample vector can be constructed as follows:

$$
A_{i j}=\left[\begin{array}{l}
A_{i j}(1,1), A_{i j}(1,2), \ldots, A_{i j}(1, C) \\
A_{i j}(2,1), A_{i j}(2,2), \ldots, A_{i j}(2, C) \\
\ldots \ldots \ldots \ldots \ldots \ldots \ldots \ldots \ldots, \ldots \ldots, \ldots \ldots \\
A_{i j}(S, 1), A_{i j}(S, 2), \ldots, A_{i j}(S, C)
\end{array}\right]
$$


Then, for an $M \times N$ digital image, each DCT coefficient corresponds to a 2-dimensional vector. There're $M \times N$ 2-dimensional vectors in all. The separability discrimination $S D(i, j)$ for the DCT coefficient at row $i$, column $j$ is calculated as follows:

Firstly, the inter-class distance $D_{b}(i, j)$ for each DCT coefficient $A_{i j}$ in all training samples is defined as

$$
D_{b}(i, j)=\sum_{c=1}^{C} \frac{1}{C}\left(\bar{A}_{i j}^{c}-\bar{A}_{i j}\right)^{2}
$$

Secondly, the inner-class distance $D_{w}(i, j)$ for each DCT coefficient $A_{i j}$ in all training samples can be gotten by

$$
D_{w}(i, j)=\sum_{c=1}^{C} \frac{1}{C} \sum_{s=1}^{S} \frac{1}{S}\left(A_{i j}(s, c)-\bar{A}_{i j}^{c}\right)^{2}
$$

where $\bar{A}_{i j}^{c}$ is the average of DCT coefficients at row $i$, column $j$ of the $\mathrm{c}_{\text {th }}$ sample, $\bar{A}_{i j}$ is the average of DCT coefficients at row $i$, column $j$ of all samples.

In terms of pattern recognition, the features with large inter-class distance and small inner-class distance are more suitable for classification. Base on the idea of Linear Discrimination Analysis (LDA), the definition of $S D$ for DCT coefficients is

$$
S D(i, j)=\frac{D_{b}(i, j)}{D_{w}(i, j)}
$$

Finally, $S D$ is assigned for each DCT coefficient. For one DCT coefficient, the larger $S D$ it has, the more it contributes to the recognition. This paper sets a threshold $K$, and keeps the DCT coefficients whose $S D$ are larger than $K$.

\section{Results and analysis}

To verify the effectiveness of our method compared with other ones, all experiments are done under an infrared face database built by ourselves with a ThermoVisionA40 infrared camera. It contains 1000 photos taken under the same condition. To be specific, there are 50 persons with 20 photos for each person. All photos are kept according to the original temperature. To check the robustness of the recognition system, there're 165 photos taken under different ambient temperature in our database. The temperature varies from $24.3^{\circ} \mathrm{C}$ to $28.4^{\circ} \mathrm{C}$. The original size for each image is $240 \times 320$. The face 


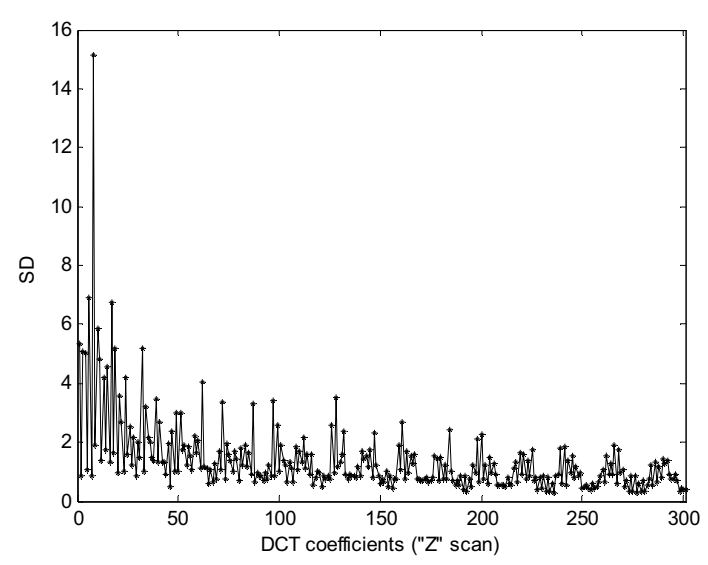

Fig. 1. SD distribution in blood perfusion image.

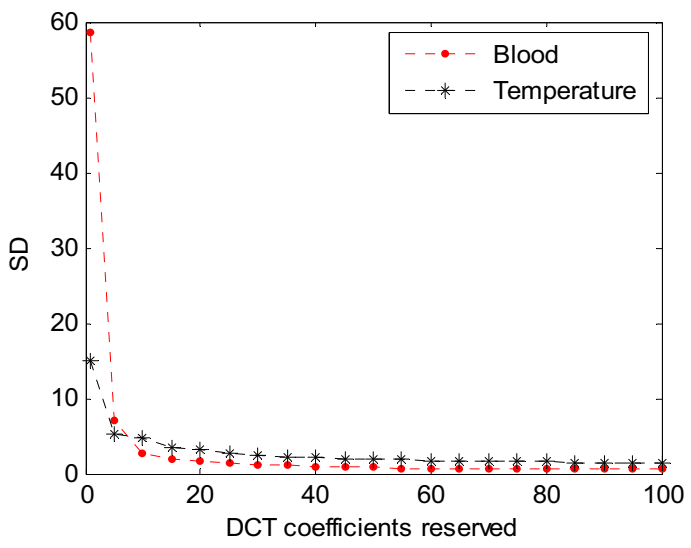

Fig. 2. Relationship between SD and DCT coefficients.

detection method is the modulation of the RIFARS system [20]. After preprocess and normalization [11], the resolution of the standard face changes into $80 \times 60$. Our experiments neglect that $\lambda$ varies from point to point and regard $\lambda$ at every point as a constant. Through estimation and repeated experiments, the best recognition performance is obtained at $\lambda=4.5 \times 10^{5}$.

\subsection{SD distribution in DCT domain}

Based on our training data under the same condition, Figure 1 demonstrates the distribution of $S D$ in DCT domain for blood perfusion images. With the increase of frequency, $S D$ for DCT coefficients fluctuate, rather than decrease monotonously. Therefore, the traditional feature selection, Z-scan, is unsuitable for selecting effective discriminative features.

In training samples, $S D$ for DCT coefficients of thermograms and blood perfusion images are descendent sorted, shown in Figure 2 (only the first $100 S D$ are listed). After the blood perfusion transformation, $S D$ for the first 5 DCT coefficients in blood perfusion images are far greater and converge much faster than those in thermograms. Therefore, blood perfusion images after DCT transform, have highlighted the contribution of features with powerful discrimination for recognition.

\subsection{Infrared face recognition experiments}

To describe the difference precisely between wrongly recognized samples and correctly recognized ones, based on Euclidean distance, this paper defines two ratios R1 and R2, where R1 denotes, under correct recognition, the average ratio of minimal distance in testing samples to the distance in the nearest wrong one. $\mathrm{R} 2$ denotes, under wrong recognition, the average ratio of minimal distance in testing samples to the distance in the nearest correct one. Theoretically, both R1 and R2 are between 0 and 1. The closer to $0 \mathrm{R} 1$ is and the closer to $1 \mathrm{R} 2$ is, the better the recognition performance will be.

Infrared face recognition experiments are tested under the same/different ambient temperature, respectively. Under the same temperature, 1000 photos are utilized, 500 of which are for training, and the other 500 for recognition. In the following results, "BP" denotes blood perfusion images obtained 
Table 1

Best recognition rates of the same-session data

\begin{tabular}{|l|l|l|l|}
\hline Method & Recognition rate & R1 & R2 \\
\hline BP & $98 \%$ & 0.30 & 0.75 \\
\hline TBP & $97.6 \%$ & 0.32 & 0.74 \\
\hline OT & $95 \%$ & 0.42 & 0.78 \\
\hline
\end{tabular}

by Pennes equation, "TBP" means traditional blood perfusion in [11] and "OT" denotes original thermograms. The results for training samples under the same temperature are shown in Table 1.

From Table 1, one can see that, compared to thermograms, discretized blood perfusion images promote the recognition performance greatly. However, compared with the model in [11], our model hardly increases the recognition rate, which is due to the fact that the ambient temperate doesn't affect the performance of infrared face recognition. Both models have extracted good biological features, and their recognition rates are higher than those of thermograms. Furthermore, our model achieves smaller R1, which shows better discrimination. Hereby, compared with thermograms, both blood perfusion models extract more stable blood perfusion information.

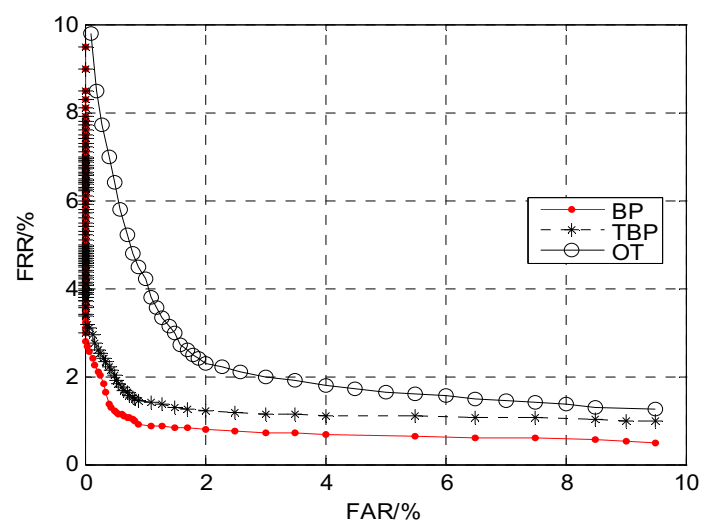

Fig. 3. ROC curves of BP, TBP and OT.

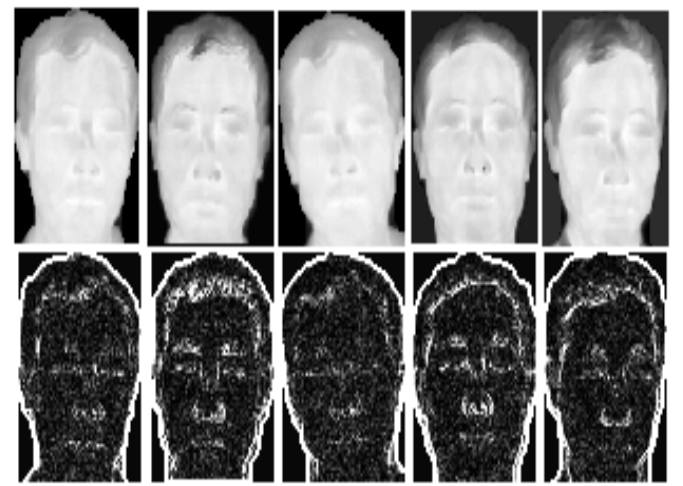

Fig. 4. Infrared face image and corresponding blood perfusion image of time-elapse data.

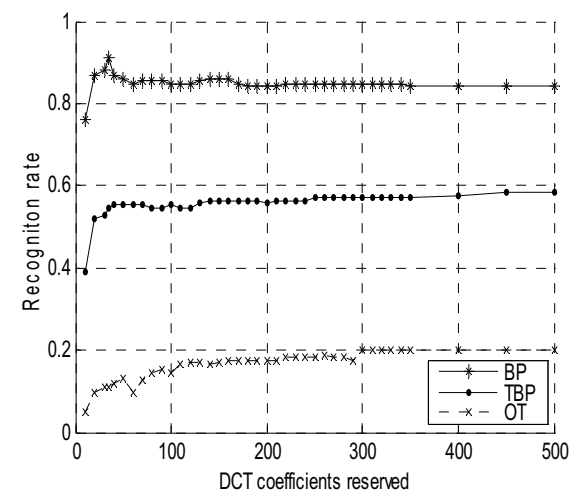

Fig. 5. Recognition rate of time-elapse data. 
Table 2

Best recognition rates of the time-elapse data

\begin{tabular}{|l|l|l|l|}
\hline Method & Recognition rate & R1 & R2 \\
\hline BP & $90.3 \%$ & 0.80 & 0.89 \\
\hline TBP & $55.6 \%$ & 0.80 & 0.77 \\
\hline OT & $26.3 \%$ & 0.91 & 0.77 \\
\hline
\end{tabular}

In the verification case, one face of each person is registered as the reference image and the rest are used as the probe ones. The receiver operating characteristic (ROC), which is a plot of the false rejection rate (FRR) versus the false acceptance rate (FAR), is simulated as shown in Figure 3. The BP methods are slightly superior to TBP method. The blood perfusion models based on BP and TBP contribute more to ROC curve than OT method.

Under varying ambient temperature, 400 images under the same temperature are chosen as the training set. The testing samples are 165 images taken under different temperature. Infrared face images and its corresponding blood perfusion images of time-elapse data are shown in Figure 4. Employing DCT and feature selection for recognition, the results are shown in Figure 5. It can be seen from Figure 5 that the proposed model outperforms the one in [11] when almost all DCT features are used for recognition. Therefore, our model possesses more stable biological features. After feature selection, when only 68 DCT coefficients are left, the recognition rate reaches the peak. Feature selection not only helps achieving higher recognition rate, but also reduces the computation complexity. 500 images under the same temperature are employed as the training samples, and 165 images under different temperature as the testing samples. After feature extraction by DCT and our feature selection, the results are shown in Table 2.

From Table 2, one can see that the recognition rates of the proposed blood perfusion are higher than those in [11]: the recognition rate rises by $34.7 \%$. The reason is that our model makes use of the heat conduction in tissues, and takes into account the relations between points in tissues (regarding adjacent temperature points relevant). It can describe facial bio-heat principle more precisely. However, the blood perfusion model in [11] keeps facial temperature points independent from each other based on thermal equilibrium, and neglects the heat conduction between tissues. As a result, under varying ambient temperature, the proposed blood perfusion images own more stable features and better recognition performance. In conclusion, the blood perfusion images obtained by our model based on Pennes equation could reduce the impact of ambient temperature on infrared face recognition, and acquire relatively stable biological features. The new method achieves better recognition performance than traditional methods under varying ambient temperature.

To verify the efficiency of feature extraction algorithm, traditional PCA+LDA and our feature extraction algorithm are tested based on our model. PCA+LDA method proposed in [21] can get the highest recognition rate of $97.4 \%$ for non-time-lapse data, and $89.6 \%$ for time-lapse data while our algorithm achieves $98 \%$ and $90.3 \%$, respectively. Therefore, our feature extraction algorithm outperforms PCA+LDA method.

\section{Conclusion}

This paper proposes an infrared facial blood perfusion model based on Pennes equation, and applies it to face recognition. The main contribution of this paper is that Pennes equation considers the 
relation between different facial temperature points, and could acquire blood perfusion images complying with human tissue heat conduction. Furthermore, combination of DCT and feature selection can extract useful discriminative features in blood perfusion rates for infrared face recognition. Experiment results show that, under different ambient temperatures, our model could reduce the impact of variable ambient temperatures on recognition, and get robust recognition performance. Our future work is to obtain more accurate blood perfusion rate by adjusting parameter $\lambda$ at different points of infrared face, and to improve the precision of our model.

\section{Acknowledgment}

This work is supported by the Natural Science Foundation of China (NO. 61201456), the Natural Science Foundation of Jiangxi Province of China (No. 20132BAB201052, N0. 20142BAB207029), the Nature Science Project of Jiangxi Science and Technology University (2013QNBJRC005, 2013ZDPYJD04).

\section{References}

[1] Hu Han, Shiguang Shan, Xilin Chen et al., A comparative study on illumination preprocessing in face recognition, Pattern Recognition 46 (2013), 1691-1699

[2] A. Selinger and D. Socolinsky, Appearance-based facial recognition using visible and thermal imagery: a comparative study, Technical Report, Equinox Corporation, 2001.

[3] D. Socolinsky and A. Selinger, A comparative analysis of face recognition performance with visible and thermal infrared imagery, Proceedings of the International Conference on Pattern Recognition (ICPR) 4 (2002), 217-222.

[4] G. Hermosilla, J. Ruiz-del-Solar, R. Verschae et al, A comparative study of thermal face recognition methods in unconstrained environments, Pattern Recognition 45 (2012), 2445-2459.

[5] S.G. Kong, J. Heo, B.R. Abidi, J. Paik and M.A. Abidi, Recent advances in visual and infrared race recognition: A review, Computer Vision and Image Understanding 97 (2005), 103-135.

[6] P. Narendra, Reference-free non-uniformity compensation for IR imaging arrays, Proceedings of the Society of PhotoOptical Instrumentation Engineers (SPIE) 252 (1980), 10-17.

[7] P. Narendra and N. Foss, Shutter less fixed pattern noise correction for infrared imaging arrays, Proceedings of the Society of Photo-Optical Instrumentation Engineers (SPIE) 282 (1981), 44-51.

[8] J. Wilder, P.J. Phillips, C. Jiang et al., Comparison of visible and infrared imagery for face recognition, Proc. 2nd Int. Conf. Automatic Face and Gesture Recognition 1 (1996), 182-187.

[9] D.A. Socolinsky and A. Selinger, Thermal face recognition over time, Proc. Int. Conf. Pattern Recognition 4 (2004), 187-190.

[10] X. Chen, P.J. Flynn and K.W. Bowyer, IR and visible light face recognition, Computer Vision and Image Understanding 99 (2005), 332-358.

[11] S.Q. Wu, W.S. Lin and S.L. Xie, Skin heat transfer model of facial thermograms and its application in face recognition, Pattern Recognition 41 (2008), 2718-2729.

[12] Zhihua Xie, Shiqian Wu, Cuiqun He et al., Infrared face recognition based on blood perfusion using bio-heat transfer mode, Proc. 2010 Chinese Conference on Pattern Recognition (CCPR) 1 (2010), 1-4.

[13] M. Akhloufi and A. Bendada, Thermal faceprint: a new thermal face signature extraction for infrared face recognition, Proc. Canadian Conf. Computer \& Robot Vision 1 (2008), 269-272.

[14] J. Liu and C.C. Wang, Biological Heat Transfer, Scientific Press, Beijing, 1997.

[15] Z.M. Hafed and M.N. Levine, Face recognition using the discrete cosine transform, International Journal of Computer Vision 43 (2001), 167-188.

[16] S. Weinbaums and L.M. Jiji, A new simplified bio-heat equation for effect of blood flow on local average tissue temperature, ASME Journal of Biomechanical Engineering 107 (1985), 131-139.

[17] J.W. Baish, P.S. Ayyaswamy and K.R. Foster, Heat transfer mechanism in vascular tissues: a model comparison, ASME Journal of Biomechanical Engineering 103 (1994), 324-331. 
[18] Yankun Zhang and Chongqing Liu, A novel face recognition method based on linear discriminant analysis, Journal of Infrared and Millimeter Waves 22 (2003), 327-330.

[19] Bae Jeongmin, Ku Bonwha, D.K. Han and Ko Hanseok, Combining infrared and visible images using novel transform and statistical information, Proc. of 2012 IEEE Ninth International Conference on Advanced Video and Signal-Based Surveillance (AVSS 2012) 1 (2012), 149-153.

[20] S.Q. Wu, L.J. Jiang, L. Cheng et al., RIFARS: a real-time infrared face recognition system, Asian Biometrics Workshop 1 (2003), 1-6.

[21] S.G. Hua, Y. Zhou and T. Liu, PCA+LDA based thermal infrared imaging face recognition, Pattern Recognition and Artificial Intelligence 21 (2008), 160-164. 